\title{
DYNAMIC LIGHT SCATTERING SIGNAL CONDITIONING FOR DATA PROCESSING
}

\author{
REI Silviu \\ Ph.D. student, Research and Development Group Leader, Faculty of Engineering, "Lucian Blaga” University of \\ Sibiu, Romania, silviu.rei@ulbsibiu.ro
}

CHICEA Dan

Professor, Faculty of Sciences, “Lucian Blaga”University of Sibiu, Romania, dan.chicea@ulbsibiu.ro

ILIE Beriliu

Lecturer, Faculty of Engineering, “Lucian Blaga”University of Sibiu, Romania, beriliu.ilie@ulbsibiu.ro

OLARU Sorin

Master Student, Production Engineer, Faculty of Sciences, “Lucian Blaga” University of Sibiu, Romania

\begin{abstract}
When performing data acquisition for a Dynamic Light Scattering experiment, one of the most important aspect is the filtering and conditioning of the electrical signal. The signal is amplified first and then fed as input for the analog digital convertor. As a result a digital time series is obtained. The frequency spectrum is computed by the logical unit offering the basis for further Dynamic Light Scattering analysis methods. This paper presents a simple setup that can accomplish the signal conditioning and conversion to a digital time series.
\end{abstract}

Key words: Dynamic Light Scattering, Signal Conditioning, Time Series.

\section{Introduction}

Nanostructured materials have several applications in biology and medicine [1], mainly for investigating and delivering useful substances to the living cells. The physical properties of the relevant nanofluids and nanoparticles are in close connection to the particle size and size distribution. Therefore, the techniques used for estimating these physical properties are of major importance.

One method for determining the average particle size for nanoparticles found in a suspension is the Dynamic Light Scattering or Photon Correlation Spectroscopy. The theoretical foundation of this method is described in various papers, [2], [3], [4] being some of them. This method is an optical procedure which uses coherent light to monitor in a fast manner the particles found in a suspension. The particles act as scattering centers and become secondary light sources when the incident coherent light interacts with them. This is a process of elastic scattering. As the incident light is coherent, the secondary waves emitted by the scattering centers are also coherent and due to interference they produce a scattering field. When placing a screen or a detector at a certain angle and distance from the sample, the interference field will have minima and maxima, randomly distributed, with the visual appearance of "boiling speckles". The intensity of light in each point of this field carries information on the phase of all the scattering centers from the active volume of the sample. The intensity of the light scattered by each center is anisotropic and depends mainly on the size and on the shape of the respective center. This information can be found in the phase function, which can be represented by various computational models [5], [6], [7].

The motion of the particles is complex, being consisted mainly on sedimentation and Brownian motion [2]. It has been shown [8] that the sedimentation velocity is few orders of magnitude smaller than the velocity of the Brownian motion, when the particles size is in the nanometer scale. As a consequence, the phase function is affected mainly be Brownian motion, and since this motion is in relation to the size of the particles via the Einstein - Stokes relation, information about the size of the particles can be extracted by studying this phase. For this, the method is recording a time series using a light detector. The light detector generates an electrical signal, converting light intensity in voltage fluctuations. The electrical signal needs to be then conditioned and prepared to be offered as input for an analog-digital converter, 
which will then offer as output a digital, numerical time series that can be processed by software for further data extraction.

This paper presents a very simple setup for signal conditioning prior to data acquisition but also a simple solution for the data digitization itself. In educational projects, as well as low budget research projects, investing in a high performance data acquisition system could be problematic and even not needed. This paper presents how the authors were able to implement a low cost acquisition system capable of converting a signal into digital time series for nanoparticles size measurements using Dynamic Light Scattering (DLS) methods.

\section{Experimental Setup}

The experimental setup used is described in Fig.1. The wavelength of the light was $633 \mathrm{~nm}$, the light source was a He-Ne laser operating continuously and the power was $10 \mathrm{~mW}$. The DLS experiment was performed at $20^{\circ} \mathrm{C}$. The cuvette-detector distance $\mathrm{D}$ was $0.20 \mathrm{~m}$ and the scattering angle $\theta$ was $90^{\circ}$. This particular value was chosen for $\theta$ because it offers a bigger range for the size of the suspended particles that are to be analyzed by DLS. More details on choosing the angle and on the algorithm used in the DLS technique are presented in [7-10].

A $10 \mathrm{~s}$ time series was recorded. The suspension consisted of burned clay nanoparticles suspended in deionized water.

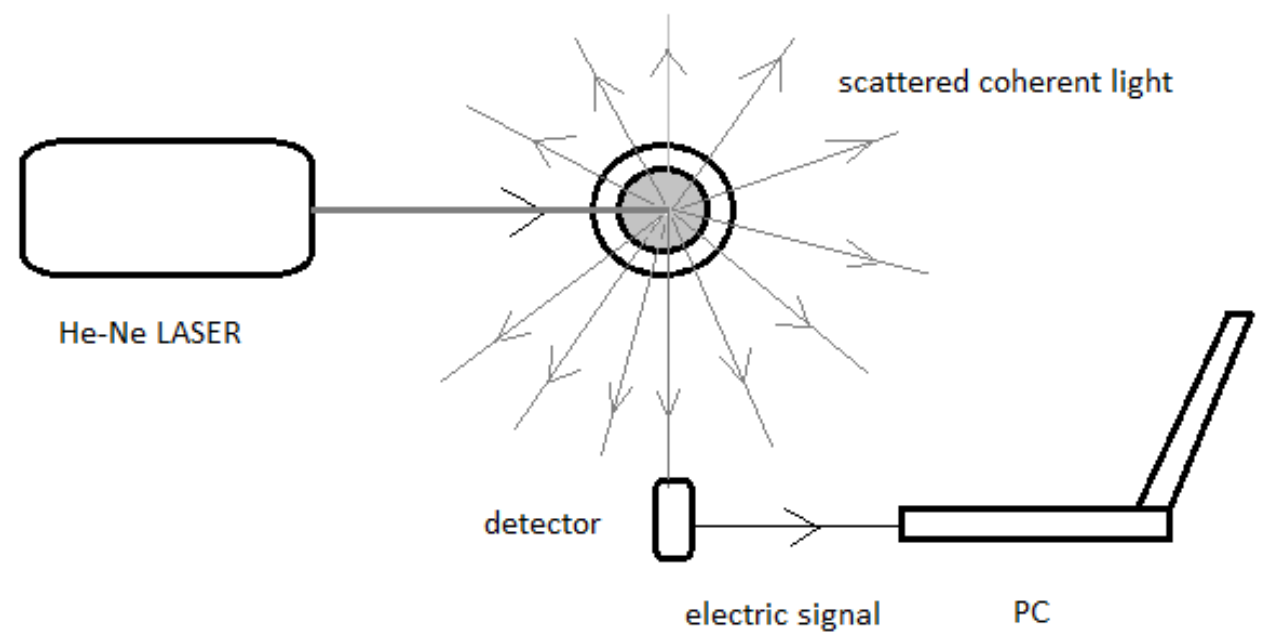

Figure 1: The DLS experimental setup

As the purpose of the work was to use an experimental setup as simple as posible, the detector we used was a phototransistor in series with a resistor, powered by a drift current source. The data acquisition was a PC sound card. The data acquisition rate was $16 \mathrm{KHz}$ and the time series was recorded in wav format.

The procedure for time series processing is presented in detail in several papers, as [8], [9], [10].

\section{Signal conditioning}

The overall process is illustrated in Fig. 2 and includes signal acquisition, signal conditioning and signal processing. 
ACTA UIVERSITATIS CIBINIENSIS - TECHNICAL SERIES

Vol. LXIX 2017

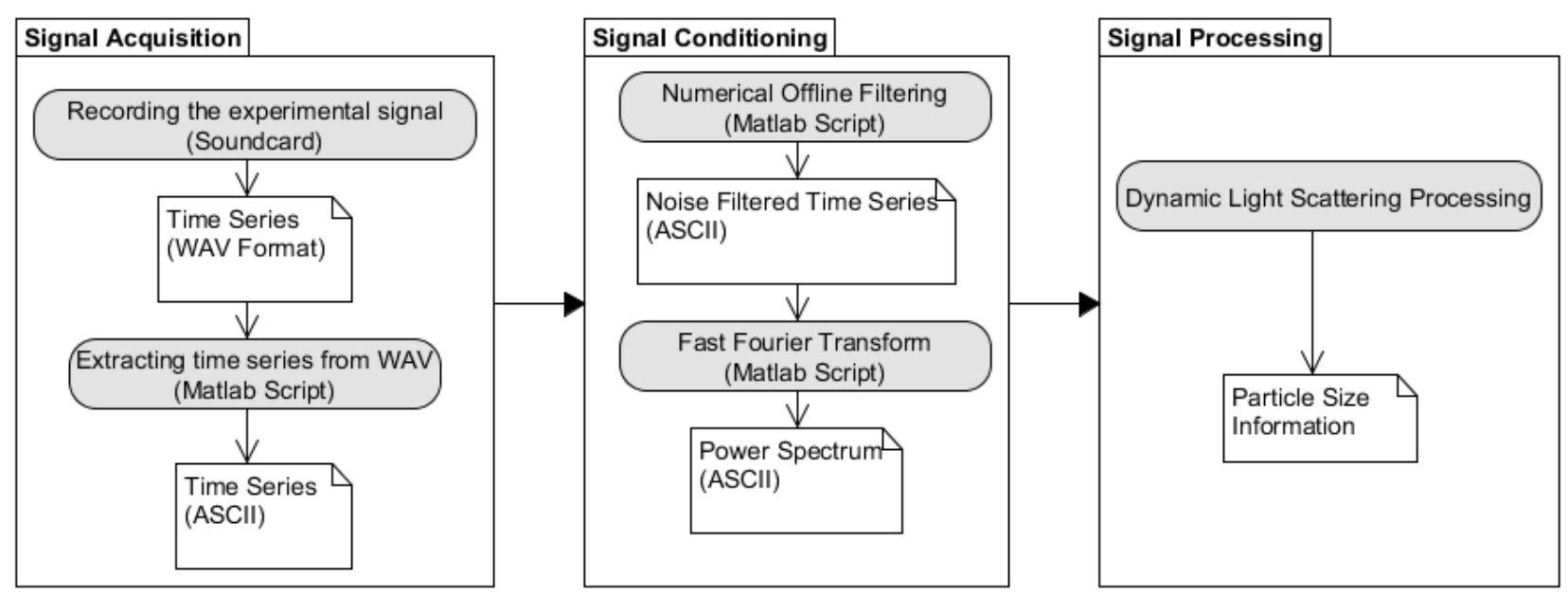

Fig. 2: The overall process including signal acquisition, signal conditioning and signal processing

The first step consists of recording and extracting the time series in digital format using a Matlab function for this purpose.

The second step in DLS time series processing consists of computing the Fourier transform of the (power) time series. Again, a Matlab function was written for this purpose. In order to produce a frequency spectrum of the recorded light intensity, also named power spectrum (PS) that can be used to fit the Lorentzian function to it, as described in detail in [8], [9], [10] and other papers, certain precautions must be taken; we called these steps signal conditioning.

A time series can last a shorter or a longer time. Consequently, the PS can contain a smaller or a bigger number of pairs frequency - amplitude. The maximum frequency in the PS is half of the frequency used in data acquisition. The longer the time series is, the bigger the density of pairs frequency-PS intensity in the above mentioned frequency range is.

The following figures will present different PSs computed using the same Matlab function, for different time series, to illustrate the effect of the number of data contained in a time series on the PS.

Fig. 3 illustrates the PS of a slice of the time series containing a number of $2^{\wedge} 10=1024$ data values. The PS contains 513 pairs of data, frequency - amplitude. We notice that the line is shattered and does not resemble a Lorentzian line, at all [8], [9], [10], making a fit of this function unrealistic.

Fig. 4 illustrates the PS of a slice of the time series containing a number of $2^{\wedge} 12=4096$ data values. The PS contains 2049 pairs of data, frequency - amplitude. We notice that the line, shattered as it is, resembles very well the Lorentizan line and this makes a fit possible and the result precise to a reasonable degree, regarding the average particle size. Moreover, we notice the $50 \mathrm{~Hz}$ line, and the harmonics, which is the electric noise induced by the power grid. 


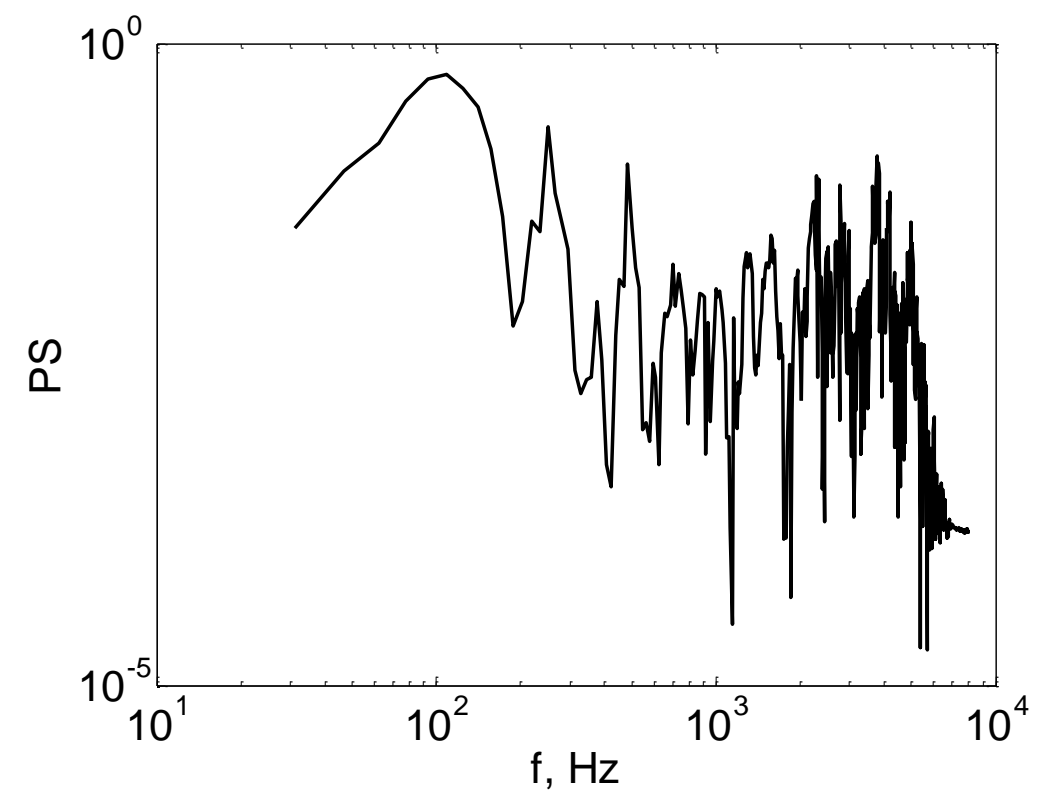

Fig 2 - The PS of a slice of the time series containing $2^{\wedge} 10=1024$ data values.

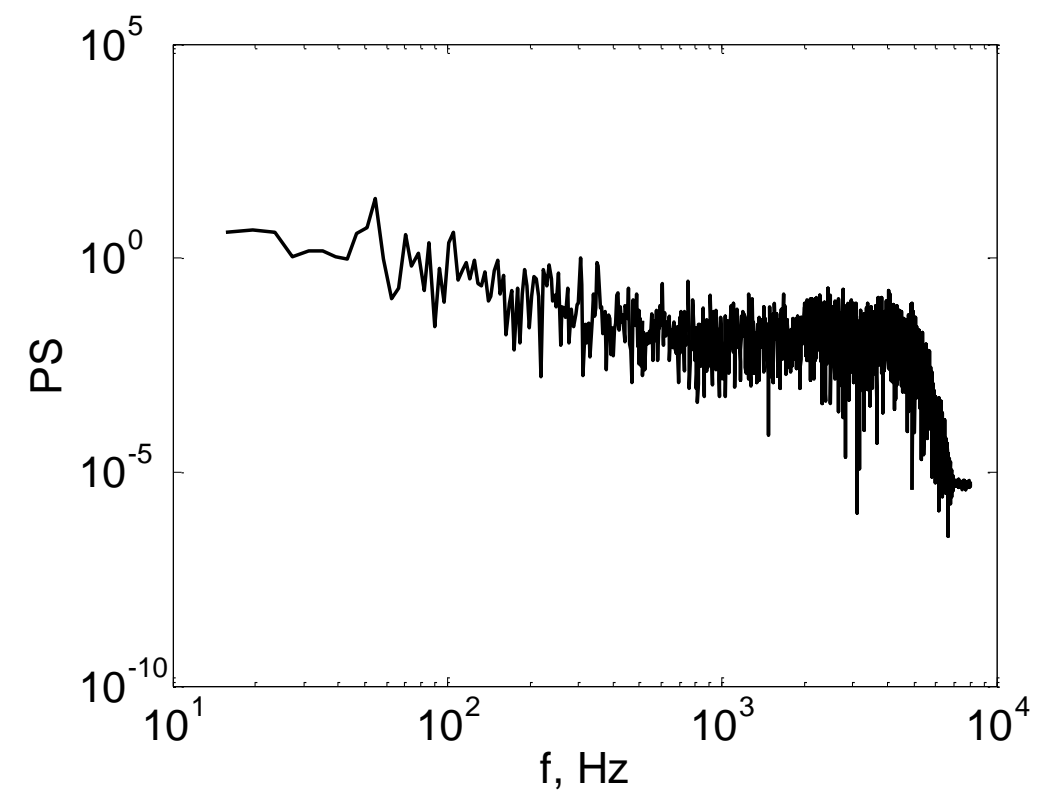

Fig 3 - The PS of a slice of the time series containing $2^{\wedge} 12=4096$ data values.

Fig. 5 depicts the PS, computed for a longer time series, counting $2^{\wedge} 17=131072$ data values. We notice that the resemblance with the Lorentzian line is much better and that the $50 \mathrm{~Hz}$ line, and the harmonics, are so evident. This suggests the third step in time series signal conditioning, which consists of filtering either the time series, or the PS to remove the $50 \mathrm{~Hz}$ line and the harmonics. The second alternative was chosen. The filtering was done using another Matlab function written for this purpose, which removes a narrow window having a controlled width centered on $50 \mathrm{~Hz}$ and on the harmonics.

Filtering in real time is not straightforward and requires active filters. The schematic of the 
experiment is intended to be as simple as possible, yet capable of producing a DLS time series that can be used in particle sizing. For this reason the schematic does not contain an active filter. Filtering is much easier once the PS is computed and consists of removing the data pairs in the PS spectrum that have the frequencies in a certain frequency window centered on a particular frequency and on the higher harmonics. Fig. 6 depicts a filtered PS, computed for a the same time series as in Fig. 5, counting $2^{\wedge} 17=$ 131072 data values but having a window of $0.5 \mathrm{~Hz}$ centered on $50 \mathrm{~Hz}$ and on the harmonics, removed.

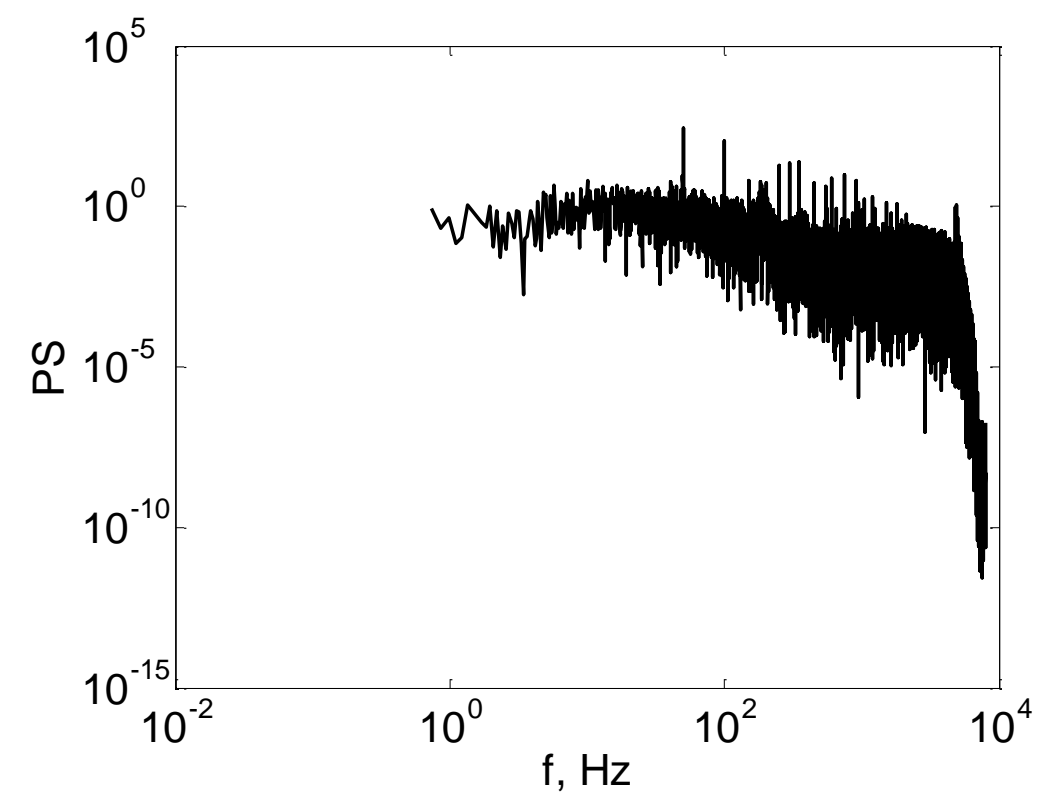

Fig 4 - The PS of a slice of the time series containing $2^{\wedge} 17=131072$ data values.

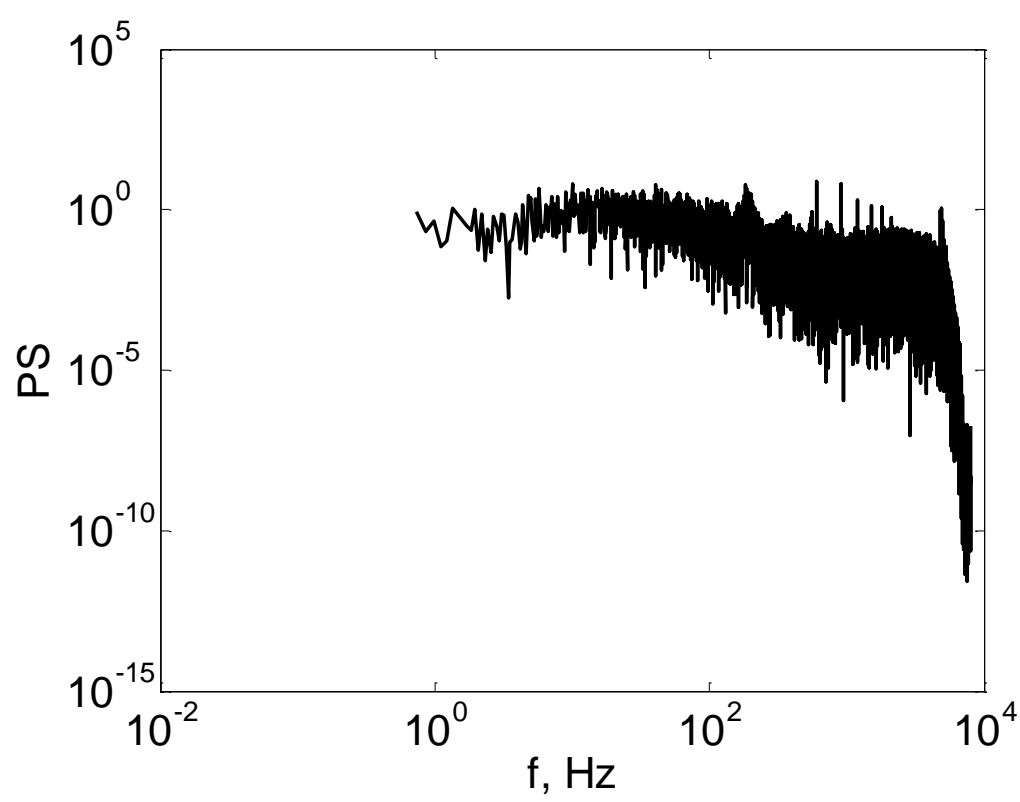

Fig 5 - The PS of a slice of the time series containing $2^{\wedge} 17=131072$ data values, filtered on 50 


\section{ACTA UIVERSITATIS CIBINIENSIS - TECHNICAL SERIES}

Vol. LXIX 2017

\section{Hz with a window of $0.5 \mathrm{~Hz}$ and on the harmonics.}

Examining Fig 5 we notice that the high amplitude lines were removed, enabling a better fit of the Lorentzian line, therefore a more precise assessment of the average particle size using the DLS technique is possible. Examples of using such a signal conditioning procedure in a DLS data processing are presented in [8], [9], [10]

\section{Conclusion}

The procedure used in signal conditioning, presented in this paper, can be used for processing time series acquired using a very simple experimental setup and a PC with a sound card. In spite of the simplicity of the data acquisition system, the signal conditioning produces a clean frequency spectrum that can be used in a DLS fitting procedure to assess the average size of the particles in a suspension. Moreover, the article indicates without a doubt that a minimum amount of data in a time series is required in order to produce a PS that illustrates the characteristics of the suspension, and the number is $2^{\wedge} 12=4096$.

Funding Information. The work presented here was partially supported by the ULBS internal research LBUS-IRG-2015-16.

\section{References}

1. O.V. Salata, "Applications of nanoparticles in biology and medicine. Journal of Nanobiotechnology, 2:3, 3-8, (2004), doi:10.1186/1477-3155-2-3.

2. J.D. Briers, "Laser Doppler, speckle and related techniques for blood perfusion mapping and imaging", Physiol. Meas. 22, R35-R66, (2001).

3. J.W. Goodman, Laser speckle and related phenomena, Vol.9 in series Topics in Applied Physics, J.C. Dainty, Ed., Springer-Verlag, Berlin, Heidelberg, New York, Tokyo, (1984).

4. M. Giglio, M. Carpineti, A. Vailati and D. Brogioli, "Near-field intensity correlations of scattered light", Applied Optics 40, 4036-4040, (2001).

5. L. Wang, S.L. Jacques, L. Zheng, "MCML - Monte Carlo modeling of light transport in multi-layered tissues",Comput Methods Programs Biomed. 47, 131-146, (1995).

6. S.A. Prahl, M. Keijer, S.L. Jacques, A.J. Welch, "A Monte Carlo Model of light propagation in tissue”, SPIE Proc. Ser. IS 5, 102-111, (1989).

7. D. Chicea, I. Turcu, "RWMCS - an alternative random walk Monte Carlo code to simulate light scattering in biological suspensions, OPTIK-International Journal for Light and Electron Optics, Vol 118/5, pp 232-236, (2007), DOI:10.1016/j.ijleo.2006.02.008

8. D. Chicea, "Coherent light scattering on nanofluids: computer simulation results", Applied Optics 47(10), 1434-1442, DOI: 10.1364/AO.47.001434.

9. D. Chicea, "Nanoparticles and Nanoparticle Aggregates Sizing by DLS and AFM", Optoelectronics and Advanced Materials - Rapid Communications vol. 4, issue 9, September 15, p. 1310 - 1315, (2010).

10. D. Chicea, E. Indrea, C. M. Cretu "Assesing Fe3O4 Nanoparticle Size By DLS, XRD and AFM", Journal of Optoelectronics and Advanced Materials Vol: 14 Issue: 5-6, pp: 460-466, (2012). 